\title{
Sex: a key consideration in understanding the etiology of psychiatric disorders and improving treatment
}

\author{
Cara Tannenbaum, MD, MSc; Patricia Boksa, PhD
}

For decades, the existence of a male or female brain was widely debated in popular culture; however, consensus has finally been reached. Data confirm, without a shadow of a doubt, that biological sex plays a critical role in brain health and disease. We have at last begun to crack the neurobiological code underlying mental health conditions, and the resulting verdict is clear: sex-related factors hold an important key to unravelling mechanisms of cerebral dysfunction.

When breakthroughs such as these occur, the curious among us like to ask, "Why now?" Does serendipity alone mark paradigm shifts in medicine? Or is luck inevitable when preparation meets opportunity?

In the case of sex-related investigations, robust attention was already being paid to male-female differences in the lifetime risk for psychiatric disorders. It was broadly recognized that there are sex differences in the prevalence, course and/or symptom profiles of many psychiatric disorders (e.g., autism, attention-deficit/hyperactivity disorder, eating disorders, schizophrenia, major depressive disorder, anxiety disorders and substance use disorders). However, the reasons for these differences are undoubtedly complex, and our understanding of the mechanisms behind them was only rudimentary.

Policies put in place during the last few years by funders such as the Canadian Institutes of Health Research and the US National Institutes of Health unquestionably accelerated the pace of mechanistic discovery by mandating that applicants outline how the analysis of both sexes will be included in experimental models where appropriate. For far too long, single-sex - usually male — cells, tissues and organisms were used as the default in basic science, owing in part to the myth that females were more variable because of their estrous cycle. ${ }^{1}$ Even when both sexes were included, few attempts were made to disaggregate the data. Push-back on funders' policies to compare both sexes was vocal at first, but resistance has waned. More researchers are aware of important discoveries concerning sex differences in normal brain development and in the pathophysiology of some psychiatric disorders, and adjust their methodological approaches to include both sexes. To build capacity, new guidelines de- scribe how to analyze sex differences in animal and clinical research through the use of more efficient study designs that examine main effects as well as sex interactions. ${ }^{2,3}$

The majority $(\sim 75 \%)$ of psychiatric disorders first emerge during adolescence and young adulthood, emphasizing the potential importance of brain developmental factors in their etiology. Substantial neuroimaging evidence from studies on children and youth now documents differences in male versus female brain structural measures, such as total brain volume, regional brain volumes, cortical thickness, white matter organization and developmental trajectories, during development. ${ }^{4}$ Interestingly, recent research indicates that microglia, the most abundant innate immune cells in the brain, appear to be sexually dimorphic during development and play an active role in both normal brain developmental processes and in brain responses to insults that are risk factors for psychiatric illness, such as perinatal infection and early life adversity. ${ }^{5}$

Neuroimaging studies also support the presence of sexdependent structural and functional alterations in the case of some psychiatric disorders. For example, many larger scale studies of autism have documented differential changes in brain neuroanatomy and in functional organization of the brain in males versus females. ${ }^{6}$ Several meta-analyses have also reported differential reductions in regional grey matter volumes in females versus males with schizophrenia. ${ }^{4}$ Sex differences in brain anatomy and function have also been observed in youth at clinical high risk for psychosis, as reported in a recent large study of 1095 youth with and without psychosis spectrum symptoms, noting sex by psychosis symptom interactions in regional brain volumes and functional striatocortical connectivity. ${ }^{7}$ While sex-dependent changes in brain volumes do not appear to be widespread in bipolar disorder (BD), a recent ENIGMA Consortium study of 1710 patients with BD and 2594 controls found a significant increase in volume of the thalamus in male but not female participants. ${ }^{8}$ In the case of major depressive disorder (MDD), a recent study of 610 community-dwelling participants found that lifetime diagnosis of MDD was associated with regional grey matter volume changes, some of which

Correspondence to: C. Tannenbaum, Centre de Recherche de l'Institut universitaire de gériatrie de Montréal, 4545 Queen Mary Road, Montreal, Quebec, Canada H3W 1W5; cara.tannenbaum@umontreal.ca

DOI: $10.1503 /$ jpn. 190165 
were common to both sexes, but others that were selective for one or the other sex. ${ }^{9}$

At a molecular level, opportunities offered by techniques such as single-cell RNA sequencing have enabled researchers to detect sex-specific qualitative differences in brain cell transcriptional responses that would not have been possible with standard multi-cell approaches. For instance, comparison of postmortem prefrontal cortices of age-matched men and women with and without Alzheimer disease revealed that increased Alzheimer pathology correlated with a global transcriptional activation in oligodendrocytes in men, but not in women. ${ }^{10}$ In contrast, women with Alzheimer pathology exhibited a global downregulation of gene activity in both excitatory and inhibitory neurons. Studies able to associate DNA methylation patterns with neural regulation pathways also yielded fruit. ${ }^{11}$ Genes exhibiting sex-biased methylation and linked to psychiatric disorders (e.g., NRXN1, NRXN2, NRXN3, FDE4A and SHANK2) were found to be enriched in synapse-related and signalling pathways, opening up new avenues of inquiry to elucidate sex differences in psychiatric disorders. The idea that disparate molecular pathways in males and females could underwrite common neuropsychiatric phenotypes rapidly gained traction. For example, in studies of depression, downregulation of the gene Dusp6 was identified in the prefrontal cortex of both human females with MDD and chronically stressed female mice, but not their male counterparts, and such downregulation was found to increase the extracellular receptor kinase signalling and pyramidal neuron excitability commonly seen in depressivelike animal models. ${ }^{12}$ These findings not only provide a framework for better understanding the molecular basis of conditions such as depression, but may also shed light on the development of sex-specific treatments. Indeed, sex differences in response to both classical and novel antidepressants have been documented. ${ }^{13}$ If personalized medicine for psychiatric illness is a desired goal, then sex differences are an obvious low-hanging fruit to explore.

In parallel to the above-mentioned molecular approaches, we have seen a steady uptake of methods to tease out whether sex effects in the brain are mediated by sex chromosomes, sex hormones or the autosomes themselves. The sex chromosomes are arguably the simplest to investigate. Females have $2 X$ chromosomes, each with 1669 genes, and males have $1 \mathrm{X}$ and $1 \mathrm{Y}$ chromosome, the latter imbued with only 426 genes. Deletion of the sex-determining Sry gene from the $\mathrm{Y}$ chromosome, or insertion of the Sry gene on the $X$ chromosome can lead to failed or successful testes development in experimental male or female animals, respectively. Known as the 4-core genotype model (XX Sry-, XX Sry+, $X Y$ Sry+, XY Sry-), this manipulation allows researchers to attribute sex differences to gonadal or chromosomal influences. Genes located on the sex chromosomes can have nongonadal-related effects as well. In experimental models of Parkinson disease, which display male susceptibility, upregulation of the Sry gene in male animals and human cultured cells has been linked to accelerated dopaminergic cell death, while downregulation reduces neurodegeneration and motor deficits. ${ }^{14}$ The causal effects of estrogens and testosterone have proven more difficult to untangle because sex hormone effects likely exist along a spectrum, rather than being dimorphic. Both types of hormones are present to varying degrees in males and females across the lifespan, are tissue specific, and affect a myriad of cellular processes. Hormone blockers and gonadectomy with subsequent supplementation are techniques that can be used in animal models to try to assess causality.

The latest focus on the autosomes is perhaps the most intriguing. According to a recent study comparing male and female transcriptomes from 12 tissue samples from each of 5 different species (dog, rat, mouse, macaque and human), it appears that $85 \%-90 \%$ of the conserved sex bias between species is related to genes located on the autosomes. ${ }^{15}$ Notably, and of relevance to basic scientists using animal models, only $12 \%$ of tissue-specific transcriptomic sex biases are transferred between species.

We know that complex traits are polygenic and multifactorial, as are most mental health conditions. Proposed models to explain sex differences for complex diseases that change over time, such as obsessive-compulsive disorder and schizophrenia, are therefore also complex. ${ }^{16,17}$ The sexdependent liability threshold model posits that males and females differ in the minimum number of environmental hits and trait-associated or disease-risk alleles required to cross the threshold for diagnosis. Other hypotheses suggest that escape from $X$-chromosome inactivation may play a role. Whereas males have only 1 copy of the $X$ chromosome, females inherit a copy from each parent, and a different $X$ chromosome is randomly silenced in every cell. In humans, sex-biased gene expression can result from $X$ inactivation escape, which has been described with approximately $23 \%$ of the genes on the $\mathrm{X}$ chromosome. ${ }^{16}$ Finally, gene by environment interactions may differ between the sexes, and this is where the concept of gender comes in.

If sex is defined in terms of biology, then gender represents social, cultural and psychological external influences on health. The term "gender identity" is used to describe an individual's sense of self as masculine, feminine, both, or non-conforming. Gender roles - familial and occupational choices and behaviours - can also cause stress, as can gender relations. Gender role stereotypes and gender identity can be especially confusing for adolescents and young adults. Thirty-five per cent of transgender adolescents attempt suicide. ${ }^{18}$ Young men experience increased rates of serious mental health issues, such as suicide, substance abuse, conduct disorder and interpersonal violence, and are less likely to engage with mental health care services than are young women, suggesting that targeted interventions and an understanding of mechanisms are needed for this group. ${ }^{19}$ Exposure to environmental insults, including stressful or negative experiences, toxins and stigma, may be driven by gender-related factors in unequal doses. In turn, a large literature has documented sex differences in response to specific stressors associated with psychopathology, such as early childhood trauma, as well as in overall stress and immune responses. ${ }^{20-23}$

There are some immediate steps that can be taken to thrust forward sex and gender science in the field of 
neurobiology and psychiatry. First, many people conflate the terms sex and gender in common vernacular as well as in publications. Specificity is required to isolate whether biological or social factors are being referred to. Sometimes interactions between sex and gender are difficult to separate, but as a general rule, inappropriate terminology should be corrected. Second, inclusion and analysis of both sexes in animal and human studies should become standard practice. ${ }^{2,3,24}$ Although the feasibility of recruiting 50:50 human participant representation for a sex-skewed disease can be challenging and not always possible, we believe researchers need to do their best to ensure that studies take into account potential sex differences in order to move toward a personalized approach in health care. Oversampling of the underrepresented sex so that the sample size will have enough power to draw statistically valid conclusions is the gold standard. In the event that it is not possible to recruit enough of the underrepresented sex to draw robust conclusions, we recommend including as many of the underrepresented sex in the study as possible, and disaggregating and presenting the raw findings by sex - or gender - so the data can be used for future meta-analyses. Finally, adjusting for sex should be avoided unless stratified analyses show sex-specific associations with both the exposure and the outcome. Disaggregating and presenting the raw findings by sex is critical for improving accuracy and avoiding misinterpretation of data.

The Journal of Psychiatry and Neuroscience encourages authors to follow the Sex and Gender Equity in Research (SAGER) guidelines, which indicate how authors should include sex and gender considerations where relevant (https:/ / jpn.ca/instructions-for-authors/). We also point readers who want to learn more to consult some of the references to this editorial, as well as the resource page and online learning modules on sex and gender in science from the Canadian Institutes of Health Research (http://www.cihr-irsc-igh-isfh. $\mathrm{ca} /$ ). These modules are now becoming mandatory for undergraduate and graduate students in various health sciences, and medical and residency programs across Canada.

A paradigm shift is occurring in the way we investigate and understand the mechanistic underpinnings of sex differences in health and disease. The years ahead are bound to witness exciting innovations in the way we approach sex and gender in relation to mental health conditions. Preparation has finally met opportunity, leading to breakthroughs and promises for the delivery of personalized therapies at the point of care.

Affiliations: From the Institute of Gender and Health, Canadian Institutes of Health Research (CIHR) and Faculties of Medicine and Pharmacy, Université de Montréal, Montreal, Que., Canada (Tannenbaum); and the Department of Psychiatry, McGill University, Montreal, Que., Canada (Boksa).

Competing interests: None declared.

\section{References}

1. Becker JB, Prendergast BJ, Liang JW. Female rats are not more variable than male rats: a meta-analysis of neuroscience studies. Biol Sex Differ 2016;7:34.

2. Beltz AM, Beery AK, Becker JB. Analysis of sex differences in preclinical and clinical data sets. Neuropsychopharmacology 2019;10.1038/ s41386-019-0524-3.

3. Buch T, Moos K, Ferreira FM, et al. Benefits of a factorial design focusing on inclusion of female and male animals in one experiment. J Mol Med 2019; 97:871-877.

4. Kaczkurkin AN, Raznahan A, Satterthwaite TD. Sex differences in the developing brain: insights from multimodal neuroimaging. Neuropsychopharmacology 2019;44:71-85.

5. Nelson LH, Saulsbery AI, Lenz KM. Small cells with big implications: Microglia and sex differences in brain development, plasticity and behavioral health. Prog Neurobiol 2019;176:103-19.

6. Lai MC, Lerch JP, Floris DL, et al. Imaging sex/gender and autism in the brain: Etiological implications. J Neurosci Res 2017;95:380-97.

7. Jacobs GR, Ameis SH, Ji JL, et al. Developmentally divergent sexual dimorphism in the cortico-striatal-thalamic-cortical psychosis risk pathway. Neuropsychopharmacology 2019;44:1649-58.

8. Hibar DP, Westlye LT, van Erp TG, et al. Subcortical volumetric abnormalities in bipolar disorder. Mol Psychiatry 2016;21:1710-6.

9. Ancelin ML, Carrière I, Artero $S$, et al. Lifetime major depression and grey-matter volume. J Psychiatry Neurosci 2019;44:45-53.

10. Mathys H, Davila-Velderrain J, Peng Z, et al. Single-cell transcriptomic analysis of Alzheimer's disease. Nature 2019;570:332-7.

11. Xia Y, Dai R, Wang K, et al. Sex-differential DNA methylation and associated regulation networks in human brain implicated in the sex-biased risks of psychiatric disorders. Mol Psychiatry 2019; 10.1038/s41380-019-0416-2.

12. Labonté B, Engmann O, Purushothaman I, et al. Sex-specific transcriptional signatures in human depression. Nat Med 2017;23:1102-11.

13. Williams AV, Trainor BC. The impact of sex as a biological variable in the search for novel antidepressants. Front Neuroendocrinol 2018;50:107-17.

14. Lee J, Pinares-Garcia P, Loke H, et al. Sex-specific neuroprotection by inhibition of the Y-chromosome gene, SRY, in experimental Parkinson's disease. Proc Natl Acad Sci U S A 2019;116:16577-82.

15. Naqvi S, Godfrey AK, Hughes JF, et al. Conservation, acquisition, and functional impact of sex-biased gene expression in mammals. Science 2019;365:eaaw7317.

16. Khramtsova EA, Davis LK, Stranger BE. The role of sex in the genomics of human complex traits. Nat Rev Genet 2019;20:173-90.

17. Khramtsova EA, Heldman R, Derks EM, et al. Sex differences in the genetic architecture of obsessive-compulsive disorder. Am J Med Genet B Neuropsychiatr Genet 2019;180:351-64.

18. Johns MM, Lowry R, Andrzejewski J, et al. Transgender identity and experiences of violence victimization, substance use, suicide risk, and sexual risk behaviors among high school students -19 states and large urban school districts, 2017. MMWR Morb Mortal Wkly Rep 2019;68:67-71.

19. Rice SM, Purcell R, McGorry PD. Adolescent and young adult male mental health: Transforming system failures into proactive models of engagement. J Adolesc Health 2018;62:S9-17.

20. Tiwari A, Gonzalez A. Biological alterations affecting risk of adult psychopathology following childhood trauma: a review of sex differences. Clin Psychol Rev 2018;66:69-79.

21. Helpman L, Zhu X, Suarez-Jimenez B, et al. Sex differences in trauma-related psychopathology: A critical review of neuroimaging literature (2014-2017). Curr Psychiatry Rep 2017;19:104.

22. Rincón-Cortés M, Herman JP, Lupien S, et al. Stress: Influence of sex, reproductive status and gender. Neurobiol Stress 2019;10:100155.

23. Rainville JR, Hodes GE. Inflaming sex differences in mood disorders. Neuropsychopharmacology 2019;44:184-99.

24. Clayton JA, Tannenbaum C. Reporting sex, gender, or both in clinical research? JAMA 2016;316:1863-4. 